\section{Foveal detachment after successful retinal reattachment for macula on rhegmatogeneous retinal detachment: an ocular coherence tomography evaluation}

SK Gibran ${ }^{1}$, A Alwitry ${ }^{1}$ and PE Cleary ${ }^{2}$
${ }^{1}$ Eye, Ear, Nose and Throat Centre, Queens Medical Centre, Nottingham, UK

${ }^{2}$ Department of Ophthalmology, Cork University Hospital, Cork, Ireland

Correspondence: SK Gibran Eye, Ear, Nose and Throat Centre,

Queens Medical Centre, Nottingham, NG7 2UH, UK Tel: + 44115924 9924; Fax: +44 1159709963 . E-mail: syedgibran@ yahoo.com

Received: 15 June 2005 Accepted: 4 August 2005 Published online: 30 September 2005

Financial/proprietary interests: None

\begin{abstract}
Purpose Foveal detachment after apparently successful retinal reattachment surgery for macula-on retinal detachments (RDs) has been previously documented. This pilot study aimed to utilize ocular coherence tomography (OCT) imaging to investigate foveal architecture after routine retinal detachment surgery and correlate this to visual acuity. Methods Prospective recruitment of patients attending one unit with macula-on RDs. Patients underwent full clinical examination including OCT preoperatively and RD surgery undertaken by scleral buckling, external drainage and air injection. Postoperatively patients had clinical examinations and OCT at 1 week, 1, 3, 6, and 12 months.

Results A total of 12 consecutive patients were recruited into the study. All had maculaon RDs and normal OCTs at onset. There were no operative or postoperative complications. Retinal reattachment was achieved in all cases within $24 \mathrm{~h}$ postoperatively. At 1 month six of 12 patients $(50 \%)$ showed foveal detachment on OCT, which was invisible on clinical examination. At 3 months, the foveal detachment persisted in four $(33 \%)$ of these patients. In these cases the foveal detachment persisted at 6 months follow-up, however, a reduction in subfoveal fluid was noted. All cases had foveal reattachment by 12 months postoperatively. Visual acuity was closely correlated to the presence of foveal attachment.
\end{abstract}

Discussion A high proportion of patients with successful retinal reattachment surgery had foveal detachments postoperatively. This phenomenon was associated with reduced visual acuity. The aetiology of this occurrence is unknown and warrants further investigation as there is the potential of a longterm effect on vision.

Eye (2006) 20, 1284-1287. doi:10.1038/sj.eye.6702098; published online 30 September 2005

Keywords: retinal detachment; foveal detachment; optical coherence tomography; macula detachment

\section{Introduction}

In retinal detachment (RD) surgery the primary concern is flattening of the retina and preservation of central vision by preventing propagation of the detachment involving the macula. When the macula is spared it is generally agreed upon and intuitively logical that surgery should be performed as soon as possible to prevent macula detachment and concurrent loss of central acuity. The presence of and duration of a macular detachment associated with RD is known to be a significant prognostic factor for visual recovery after successful RD surgery. ${ }^{1,2}$

Postoperative pathology may also affect final visual acuity including the development of macular pucker, pigmentary dispersion, ${ }^{3}$ macular retinal folds ${ }^{4}$ and subretinal lesions. ${ }^{5}$ 
Despite early operative intervention in patients with clinically macula-on RDs postoperative foveal detachment has been seen. ${ }^{6}$ This foveal detachment may have an impact on final visual acuity after successful surgery and is the focus of the present study.

The purpose of this prospective observational study is to evaluate the pre- and postoperative foveal architecture by ocular coherence tomography (OCT) after successful surgery for macula on rhegmatoneous RD (Figure 1).

\section{Methods}

All patients presenting to our service between the dates of June 2003 and December 2003 were considered for recruitment into the study.

Inclusion criteria were the presence of a unilateral RD, which was suitable for repair with scleral buckling, external drainage and air injection (DACE). All patients had spared macula preoperatively as assessed by slit-lamp indirect ophthalmoscopy by an experienced vitreoretinal surgeon. In addition, the most posterior extension of the detachment had to be further than three disc diameters from the fovea. Exclusion criteria were the presence of any other ocular or macular pathology.

Demographic data were collected on the patient and suitability for entry into the study, with particular

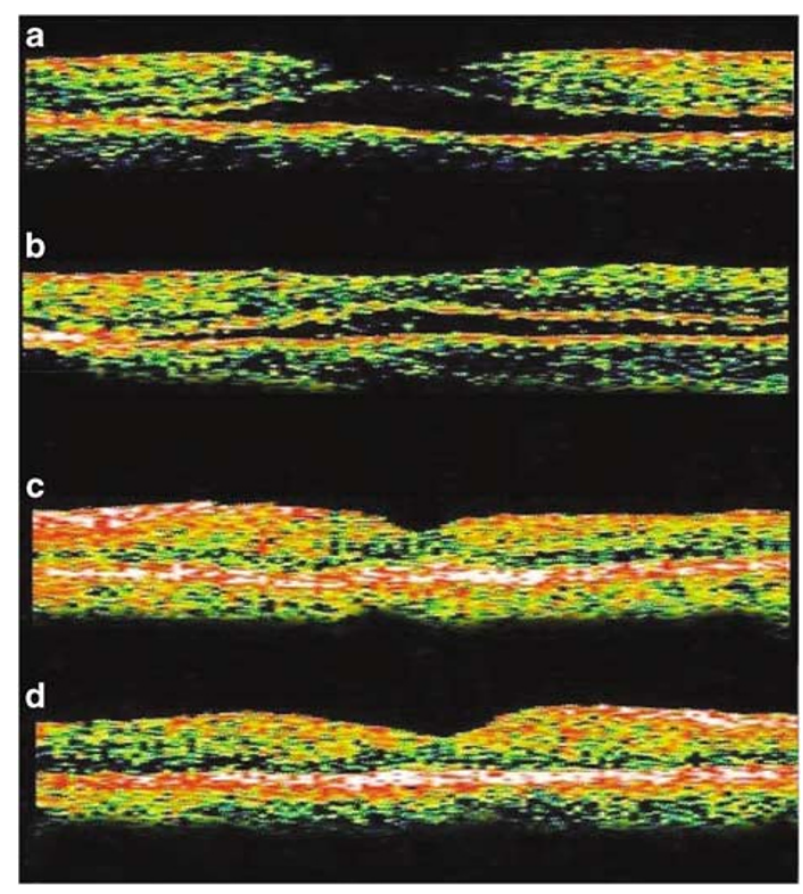

Figure 1 OCT images obtained from patient 7 (superotemporal retinal detachment with the extent $>5$ disc diameter away from fovea, BCVA $6 / 6$ preoperatively) (a) 1-month postoperatively, BCVA 6/18. (b) 3 months postoperatively, BCVA 6/18. (c) 6 months postoperatively, BCVA 6/12. (d) 12 months postoperatively, BCVA 6/9. reference to the clinical state of the macula, was confirmed by another of the authors.

All patients had complete clinical examination preoperatively including best corrected Snellen visual acuity (BCVA), slit-lamp biomicroscopic indirect ophthalmoscopy with 78D lens and OCT (6 radial lines, OCT 2, Carl Zeiss Meditec AG, Germany).

Informed consent for the examination and surgery was obtained from each patient after full explanation of the procedure. All operations were performed under general anaesthesia by two of the authors (SKG and PEC). Scleral buckling was performed in the sequence of DACE. External drainage of subretinal fluid was performed with 25-gauge needle and air was injected in opposite quadrant with a same gauge needle. The number of cryopexy applications utilized was three to five in all patients. A circumferential 277 scleral explant with two to four scleral sutures were used in all cases. All operations were uncomplicated. Postoperatively only subconjunctival antibiotics were used and patients were postured in a lateral position for the following 5 days.

Patients were examined at 1 week, 1, 3, 6, and 12 months postoperatively with complete ophthalmic examination. An OCT was performed at 1, 3, 6, and 12 months.

\section{Results}

In all, 12 consecutive patients were recruited into the study. The average age of the patients was 42 years (range 21-58 years). The period of evolution of symptoms and presentation to hospital ranged from 1 to 3 days. All patients were operated on within $12 \mathrm{~h}$ of presentation at the hospital. All patients had normal OCTs on presentation. All patients were phakic. The age/sex, extent of RD and status of posterior hyaloid face is shown in Table 1.

In all patients, the RD was caused by one horseshoe retinal tear and an additional retinal hole was found in two patients.

Retinal reattachment was achieved in all cases within $24 \mathrm{~h}$ postoperatively. At 1 month six of 12 patients (50\%) showed foveal detachment on OCT, which was invisible on clinical examination.

At 3 months, the foveal detachment persisted in four (33\%) of these patients. In these cases the foveal detachment persisted at 6 months follow-up, however, a reduction in subfoveal fluid was noted. All cases had foveal reattachment by 12 months postoperatively.

The BCVAs and OCT state of the fovea at each followup is shown for those patients who had foveal detachment is shown in Table 2. 


\section{Discussion}

The cause of foveal detachment in patients with an uninvolved macula preoperatively after successful and uncomplicated scleral buckling is not clear. Provided that all retinal breaks are treated successfully peroperatively, the source of subfoveal fluid should not be via the retinal break and thus remains speculative. This fluid may represent leakage from the retinal pigmentary epithelium (RPE) and/or choroidal blood vessels due to alteration in the blood-retinal barrier, however, Wolfensberger and Gonvers ${ }^{7}$ showed with postoperative fluorescein angiography that there was no evidence of vascular leakage in such cases.

Scleral buckling has been described to affect both subfoveal choroidal ${ }^{8}$ and retinal blood flow. ${ }^{9}$ These haemodynamic changes may alter the polarity of the RPE and lead to fluid leakage. Conversely, inflammation induced by scleral buckling itself may be the source of the subfoveal fluid. ${ }^{10,11}$ It is possible that the isolated area of subfoveal fluid could be the result of fluid movement from the original location of the RD towards the posterior pole during the surgery or afterwards. Theodossiadis et $a l^{6}$ speculated that the proximity of the RD to the fovea

Table 1 Patients profile

Pt Age/sex $\begin{gathered}\text { Duration of Eye Location of RD Clock (h) PVD } \\ \text { symptoms (D) }\end{gathered}$

\begin{tabular}{rllllll}
\hline 1 & $40 / \mathrm{M}$ & 3 & $\mathrm{R}$ & Superotemporal & $0900-1200$ & - \\
2 & $47 / \mathrm{M}$ & 1 & $\mathrm{R}$ & Temporal & $0700-1200$ & - \\
3 & $37 / \mathrm{M}$ & 2 & $\mathrm{R}$ & Superior & $1000-1300$ & + \\
4 & $57 / \mathrm{M}$ & 1 & $\mathrm{R}$ & Superior & $1000-1400$ & - \\
5 & $52 / \mathrm{F}$ & 3 & $\mathrm{R}$ & Superior & $0900-1200$ & - \\
6 & $21 / \mathrm{M}$ & 2 & $\mathrm{R}$ & Temporal & $0700-1200$ & + \\
7 & $34 / \mathrm{M}$ & 2 & $\mathrm{~L}$ & Superotemporal & $1200-1600$ & + \\
8 & $47 / \mathrm{F}$ & 1 & $\mathrm{R}$ & Superonasal & $1100-1500$ & - \\
9 & $29 / \mathrm{M}$ & 2 & $\mathrm{~L}$ & Superior & $1000-1400$ & + \\
10 & $50 / \mathrm{F}$ & 3 & $\mathrm{R}$ & Nasal & $1300-1700$ & - \\
11 & $42 / \mathrm{M}$ & 2 & $\mathrm{~L}$ & Superotemporal & $0900-1300$ & - \\
12 & $58 / \mathrm{M}$ & 2 & $\mathrm{~L}$ & Superior & $1000-1400$ & - \\
\hline
\end{tabular}

Pt, patient; $\mathrm{D}$, days; RD retinal detachment; $\mathrm{h}$, hours; PVD, posterior vitreous detachment. was indeed the cause of this occurrence as in two out of three of their patients with postoperative submacular fluid, the posterior border of RD was within the range of 1.5-3.5 DD of fixation. In all of our patients however, the posterior border of RD was more than $3 \mathrm{DD}$ away from the fovea and thus this aetiology seems unlikely.

Another cause of fluid accumulation in the macula, which is more plausible, could be a localized loss of adherence of the RPE to Bruch's membrane (BM) due to the surgical, trauma-induced, inflammation. In this case, the disturbance of the adherence of RPE and BM would theoretically permit access of fluid from choroid to the subretinal space.

It also remains unclear if the remaining posterior hyaloid face plays a role in maintaining this serous elevation of fovea in the nonvitrectomy surgical approach to dealing with these patients. These histological features of foveal detachment and biochemical analysis of residual subfoveal fluid cannot be studied further because of the difficulty of obtaining specimens. In our series four out of the six cases with foveal detachment had an attached posterior hyaloid face.

Half of the patients examined in our case series had significant foveal detachments on OCT assessment. Interestingly, the detrimental effect on central acuity was clearly demonstrated in the majority of our cases with improvement in acuity being seen with resolution of the subfoveal fluid (Table 2). This would suggest that this is indeed a pathological process which has the theoretical potential of resulting in deleterious long-term sequelae.

Although the small numbers in our study precluded meaningful statistical analysis it appeared that there was no correlation between development of subfoveal fluid and extent/location of RD or any other patient demographics. Drainage of subretinal fluid and injection of air to seal the retinal break did not seem to prevent the postoperative foveal detachment, although the patients in our study were not postured prone after the surgery.

In cases with foveal detachment, visual acuity decreases correspondingly while the detachment is

Table 2 Pre- and postoperative VA and OCT

\begin{tabular}{|c|c|c|c|c|c|c|c|c|c|}
\hline \multirow[t]{2}{*}{ Pt } & \multirow[t]{2}{*}{ Preop VA } & \multicolumn{4}{|c|}{ Postop VA (months) } & \multicolumn{4}{|c|}{ Postop foveal OCT findings (month) } \\
\hline & & 1 & 3 & 6 & 12 & 1 & 3 & 6 & 12 \\
\hline 1 & $6 / 6$ & $6 / 9$ & $6 / 9$ & $6 / 9$ & $6 / 6$ & + & - & - & - \\
\hline 2 & $6 / 6$ & $6 / 18$ & $6 / 18$ & $6 / 12$ & $6 / 9$ & + & + & + & - \\
\hline 3 & $6 / 6$ & $6 / 12$ & $6 / 12$ & $6 / 12$ & $6 / 9$ & + & + & + & - \\
\hline 4 & $6 / 6$ & $6 / 12$ & $6 / 12$ & $6 / 12$ & $6 / 9$ & + & + & + & - \\
\hline 5 & $6 / 9$ & $6 / 12$ & $6 / 12$ & $6 / 12$ & $6 / 12$ & + & + & + & - \\
\hline 6 & $6 / 5$ & $6 / 12$ & $6 / 9$ & $6 / 9$ & $6 / 6$ & + & - & - & - \\
\hline
\end{tabular}

Pt, patient; VA, visual acuity; OCT, ocular coherence tomography. 
present. It is logical to presume that duration of postoperative foveal detachment may play a role in patients whose vision takes longer to improve. The chronicity of foveal detachment may predispose to photoreceptor damage and explain cases of incomplete postoperative visual recovery after successful RD repair following scleral buckling. Despite apparent recovery of central acuity with time it is clear in modern ophthalmology that BCVA is not a definitive marker of success. Other visual parameters, such as contrast sensitivity, for example, may be adversely affected by prolonged foveal detachment despite apparent restoration of premorbid acuity. The correlation of the postoperative foveal detachment to other visual parameters may be an avenue for further investigation.

Since clinical examination alone may fail to reveal the presence of foveal detachment the authors suggest that OCT is the tool of choice in investigating the delayed postoperative visual recovery in such cases, however, the role of potential interventions to speed visual recovery remains speculative and will only become apparent with further study.

The most crucial question is how to prevent the postoperative foveal detachment in these cases as it unlikely to be a completely innocuous phenomenon. We acknowledge that our pilot study does not answer the question of aetiology. Further research is required to assess whether the presence of this pathological process does indeed predispose to an, albeit small, detrimental effect on final outcome with regards visual acuity or visual performance as a whole. Certainly, further work should be undertaken to determine whether the onset and duration of this foveal detachment may be modified with measures such as postoperative prone posturing in these patients or the use of steroids to prevent any inflammatory component to the pathogenesis.

\section{References}

1 Burton TC. Recovery of visual acuity after retinal detachment involving the macula. Trans Am Ophthalmol Soc 1982; 80: 475-497.

2 Ross WH, Kozy DW. Visual recovery in macula-off rhegmatogenous retinal detachments. Ophthalmology 1998; 105: 2149-2153.

3 Cleary PE, Leaver PK. Macular abnormalities in the redetached retina. Br J Ophthalmol 1978; 62: 595-603.

4 Sabates NR, Sabates FN, Sabates R, Lee KY, Ziemianski MC. Macular changes after retinal detachment surgery. Am J Ophthalmol 1989; 108: 22-29.

5 Kaga T, Fonseca RA, Dantas MA, Yannuzzi LA, Spaide RF. Optical coherence tomography of bleb-like subretinal lesions after retinal reattachment surgery. Am J Ophthalmol 2001; 132: 120-121.

6 Theodossiadis PG, Georgalas IG, Emfietzoglou J et al. Optical coherence tomography findings in the macula after treatment of rhegmatogenous retinal detachments with spared macula preoperatively. Retina 2003; 23: 69-75.

7 Wolfensberger TJ, Gonvers M. Optical coherence tomography in the elevation of incomplete visual recovery after macula-off retinal detachments. Graefes Arch Clin Exp Ophthalmol 2002; 240: 85-89.

8 Movaffaghy A, Pharmakakis NM, Chamot SR, Katsimpris JJ, Pournaras JA, Pournaras CJ. Effects of squatting on subfoveal blood flow defect in pseudophakic eyes operated by cerclage. Klin Monatsbl Augenheilkd 2001; 218: 323-326.

9 Regillo CD, Sergott RC, Brown GC. Successful scleral buckling procedures decrease central retinal artery blood flow velocity. Ophthalmology 1993; 100: 1044-1049.

10 D'Hermies F, Korobelnik JF, Chauvaud D, Pouliquen Y, Parel JM, Renard G. Scleral and episcleral histological changes related to encircling explants in 20 eyes. Acta Ophthalmol Scand 1999; 77: 279-285.

11 Birgul T, Vidic B, El-Shabrawi Y. Intrusion of an encircling buckle after retinal detachment surgery. Am J Ophthalmol 2003; 136: 942-944. 